\title{
Psychosomatic consultation in the workplace: do we reach different users by changing the context?
}

\author{
Eva Rothermund ${ }^{1 *}$, Reinhold Kilian², Michael Hoelzer ${ }^{3}$, Monika Annemarie Rieger ${ }^{4}$, Harald Guendel ${ }^{1}$ \\ From Health Services Research: Evidence-based practice \\ London, UK. 1-3 July 2014
}

\section{Background}

In Germany, the proportion of mental health diagnoses in early retirement is currently at $40 \%$, constituting the largest diagnostic group. Due to demographic changes, shortage of qualified staff is an increasing challenge for social insurance funds and the labour market. Work-related stress is known to promote common mental disorders (CMD) like depression, anxiety, functional somatoform disorder or adjustment disorders and results in a declining quality of life and work performance. Although the landscape of mental health services is well established in Germany, only $40 \%$ of affected individuals managed to obtain professional care. The unmet need for easily accessible and early interventions and the economic impact of CMD led to the development of a variety of offers at the interface between company supported and conventional mental health care (CAU) e.g. the "psychosomatic consultation in the workplace" (PCIW). To learn more about this complex system we set out to analyse user profile and change 12-weeks after consultation.

\section{Materials and methods}

Observational cross-sectional design, followed by a prepost- test 12 weeks after initial consultation. By latent class analysis (LCA) individuals were classified into distinct groups based on individual response patterns. For pre-post comparison we performed variance analysis with repeated measurements. Data were collected by self-administered questionnaires: work ability (work ability index, WAI), quality of life (SF-12), mental health (PHQ9-depression, PHQ-15-somatization, PHQ-7-anxiety) and work-related stress (irritation scale, maslach burnout inventory).

'University Clinic of Psychosomatic Medicine and Psychotherapy, Ulm, Germany

Full list of author information is available at the end of the article

\section{Results}

Preliminary sample description: $\mathrm{N}=352$ individuals: PCIW $n=173 /$ CAU $n=179$. Demographic variables that differed between the groups $(\mathrm{p}<0.05)$ were age in years (PCI W44.9, SD10.1 /CAU 39.4, SD11.9), gender (PCIW $70 \%$ male/CAU $30 \%$ male), symptom duration in months (median PCIW 12 /CAU 24) and service utilization, i.e. previous contact with the psychotherapeuticpsychosomatic-psychiatric health care system (PCIW $38 \% /$ CAU 63\%). A 4-class solution was chosen due to best fit indices. Four subgroups (classes) of users with different patterns of impairment were identified; generally those with less impairment were seen in the vocational context. Pre-post test showed improvement in both groups for WAI (PCIW 30.25, SD 8.25; CAU 26.57 SD 8.86), depression, anxiety, SF-12 mental health and irritation. There was no difference between groups (PCIW vs. CAU), nor a group time effect.

\section{Conclusion}

PCIW stands for an easy accessible therapeutic offer in the vocational context. Our data suggest that we reach a different type of user. Even though the user profile differs the effect of the intervention seems to be similar.

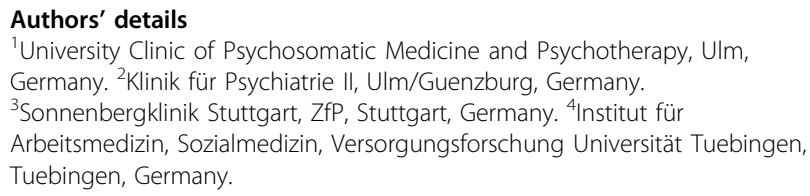

Published: 7 July 2014

doi:10.1186/1472-6963-14-S2-P105

Cite this article as: Rothermund et al:: Psychosomatic consultation in the workplace: do we reach different users by changing the context? BMC Health Services Research 2014 14(Suppl 2):P105.

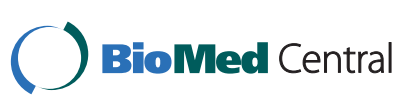

(c) 2014 Rothermund et al; licensee BioMed Central Ltd. This is an Open Access article distributed under the terms of the Creative Commons Attribution License (http://creativecommons.org/licenses/by/4.0), which permits unrestricted use, distribution, and reproduction in any medium, provided the original work is properly cited. The Creative Commons Public Domain Dedication waiver (http://creativecommons.org/publicdomain/zero/1.0/) applies to the data made available in this article, unless otherwise stated. 